\title{
Marco textual y desarrollo de la comprensión de textos académicos en inglés
}

\author{
Textual Framework and Development \\ of Academic Texts in English
}

Citation/ Para citar este Artículo: Jiménez, P., Rosas, M., \& Sáez, C. (2017). Marco Textual y desarrollo de la comprensión de textos académicos en inglés. Colomb. Appl. Linguist. J., 19(1), pp. 84-102.

Received: 05-Nov-2014 / Accepted: 26-Jun-2016

DOI: http://dx.doi.org/10.14483/calj.v19n1.7783

\section{Resumen}

Este estudio de diseño cuasi-experimental usó muestras naturalmente formadas por tres cursos regulares de primer año de una universidad chilena. El grupo experimental estuvo formado por alumnos de Ingeniería en Acuicultura y los grupos control por alumnos de Biología Marina e Ingeniería Ambiental. Nuestros objetivos fueron describir los factores que inciden en la complejidad de textos académicos escritos en inglés de Acuicultura; determinar la efectividad de un modelo de enseñanza aprendizaje basado en actividades cognitivas (estrategias) desarrolladas a partir del análisis de artículos científicos en la disciplina; comparar y analizar las estrategias de comprensión de textos disciplinares escritos en inglés desarrolladas por los alumnos del grupo experimental y los grupos control después de la intervención pedagógica. Los instrumentos cualitativos incluyeron un cuestionario y un focus group para indagar sobre las creencias de los estudiantes respecto de estrategias de comprensión de textos escritos en L1 y L2. Como resultado, identificamos los factores que inciden en la complejidad de los textos disciplinares en inglés de Acuicultura, detectamos las dificultades en la lectura de estos textos y comprobamos un desarrollo significativo de competencias lectoras en el grupo experimental al lograr un promedio mayor que los otros dos grupos control en el post-test.

Palabras clave: desarrollo de estrategias de comprensión, marco textual, textos disciplinares escritos en inglés.

\section{Abstract}

This quasi-experimental study used samples naturally formed by three first year regular courses of a Chilean university. The experimental group included Aquaculture Engineer students and the control groups included Marine Biology and Environmental Engineer students. Our aims were to describe the elements that have an impact in the complexity of academic English texts in the Aquaculture area; to determine the effectiveness of a teaching learning model using cognitive activities (strategies) based on the analysis of scientific articles in this discipline; and finally to compare and analyse the reading comprehension strategies of academic texts written in English developed by the experimental and control groups following the intervention period. With this aim in mind we elaborated the text framework concept. We used quantitative and qualitative methodology to gather data. The quantitative instrument-a pre/post test-measured

\footnotetext{
1 Universidad de Los Lagos. Osorno, Chile. rosas262000@yahoo.co

2 Universidad de Los Lagos. Chile. pablo_jimenez77@hotmail.com

3 Universidad de Los Lagos. Chile.csaez@ulagos.cl
} 
the improvement achieved by the experimental group in a 36-hour intervention period. The qualitative instruments included a questionnaire and a focus group to find out about the students' beliefs about written text comprehension strategies in L1 and L2. As a result, we identified the factors that determine the complexity of Aquaculture academic texts, we detected the students' difficulties when reading academic English texts and confirmed that the experimental group achieved a better development of reading comprehension strategies than the control groups when comparing both tests.

Keywords: academic texts written in English, reading comprehension strategy development, text framework.

\section{Introducción}

En los últimos años, como se explica a continuación, se evidencia un grupo reducido de estudios realizados en Chile, que den cuenta sobre el desarrollo de la habilidad de comprensión de textos escritos en lengua extranjera en alguno de los tres niveles del sistema educacional chileno. Respecto de investigaciones en la enseñanza superior relativas a este tema, encontramos los estudios de lbañez, (2007, 2008); Arancibia, (2010) y Lagos (2011). Los artículos acerca de comprensión de textos disciplinares escritos en inglés de Ibañez (2007, 2008) se circunscriben en el ámbito del Inglés para Propósitos Académicos (IPA), al cual pertenece este estudio.

El propósito del estudio de Arancibia (2010), realizado con estudiantes de Medicina de segundo año, fue establecer relaciones entre la cantidad y calidad del conocimiento léxico y la habilidad de comprensión de lectura. Arancibia descubrió que la relación más significativa se dio entre la cantidad de vocabulario en el nivel de 3000 palabras y la comprensión lectora. Concerniente a la calidad del conocimiento léxico y la comprensión de lectura, los resultados mostraron que ambas están relativamente asociadas; es decir, los estudiantes con mayor comprensión lectora poseen un conocimiento léxico de mayor calidad. De otro lado, Lagos (2011) describe la metodología de trabajo de cursos de ESP realizados con tres programas académicos de pregrado de su universidad. Este modelo de trabajo considera tanto las características de los alumnos como el tiempo otorgado para el logro de los objetivos planteados en los programas de los cursos. No obstante el poco tiempo asignado, y a pesar de las malas condiciones de entrada de los alumnos, el equipo de docentes de ESP ha logrado mejorar considerablemente los resultados finales desde que pusiera en práctica su modelo en todos estos cursos.

Por otra parte, el estudio de Sanhueza y Burdiles (2012) realizado con estudiantes de enseñanza secundaria da cuenta sobre las estrategias de aprendizaje y desarrollo de competencias comunicativas concernientes a habilidades receptivas y de producción, de una muestra de quince alumnos de un colegio particular bilingüe inglés-español perteneciente a un universo de colegios del mismo tipo que rindieron en Chile el examen FCE, en el año 2003. Según las autoras, en la sección de comprensión lectora de la prueba FCE, el 66,6\% de la muestra estudiada obtuvo un porcentaje de logro entre $60 \%$ a $74 \%$, un porcentaje de aprobación mínimo. Se considera poco exitoso ya que los sujetos demostraron tener niveles deficitarios en la determinación de ideas generales y específicas, en la identificación de estructuras textuales y en el establecimiento de inferencias. Al parecer, se privilegia la fluidez en vez de la precisión en el proceso de enseñanza de un idioma extranjero que dicho colegio identifica como un enfoque comunicativo, lo cual no garantiza un alto nivel de competencia comunicativa en aspectos como comprensión y producción escrita y uso del idioma (Sanhueza y Burdiles, 2012).

Desde una perspectiva universitaria, uno de los grandes problemas que los alumnos provenientes de colegios públicos en Chile enfrentan al llegar a la universidad es la lectura de artículos de su especialidad en una lengua extranjera, sobre todo en las carreras científicas. De acuerdo con los últimos resultados de la prueba nacional SIMCE Inglés (Sistema de Medición de la Calidad de la Educación), el Ministerio de Educación de Chile estimó que desde 2010 a 2014, el universo de estudiantes que certifican un nivel de inglés A2 y B1 de acuerdo con el Marco de Referencia Común Europeo, CEFR (Agencia de Calidad, 2015) subió de $11 \%$ en 2010 a $18 \%$ en 2012 y a $25 \%$ en 2014 , dándose el mayor 
aumento de estudiantes certificados en los sectores medio y medio alto. De ahí, la necesidad de incluir módulos de inglés para desarrollar la comprensión de textos escritos durante los primeros años de las carreras universitarias de universidades estatales, donde la mayoría de los estudiantes provienen de sectores bajo y medio bajo. Sin embargo, aunque los alumnos tienen dos y tres semestres de inglés, no logran leer comprensivamente artículos científicos en su especialidad. Por esa razón, decidimos aplicar una nueva metodología de enseñanza. Fue prioritario determinar la factibilidad de un modelo de enseñanza aprendizaje basado en actividades cognitivas desarrolladas a partir del análisis de artículos científicos del área de Acuicultura, con el fin de aumentar el umbral lingüístico de alumnos novatos para optimizar sus procesos de comprensión de dichos artículos.

Con este propósito, tuvimos que precisar y especificar conceptos cuya finalidad fue delimitar las áreas a intervenir e identificar unidades y relaciones propias de un texto de especialidad. Uno de ellos es la "zona de desarrollo próximo" (Vigotsky, 1988) que adquiere mayor poder explicativo al facilitar la identificación del conocimiento básico de los alumnos al momento de comenzar y finalizar la intervención y comparar el avance logrado con los grupos control. Otro concepto relevante es el de "umbral lingüístico" (Koda, 2005), definido como aquello que un alumno necesita saber lingüísticamente para poder leer comprensivamente textos en inglés. La explicación, asimismo, de los conceptos de "procesamiento" y de "estrategias" (Oxford, 1990; Gu, 2005; Grabe, 2009) fue un apoyo primordial para los objetivos de esta investigación, ya que en cuanto los alumnos conocen estos conceptos, mejoran notablemente sus capacidades lectoras. De este modo pueden analizar, reflexionar y monitorear sus procesos, reforzando sus habilidades de pensamiento superior.

Usamos un diseño cuasi-experimental con muestras naturalmente formadas por tres cursos regulares de primer año de una universidad del sur de Chile. El grupo experimental estuvo formado por la carrera de Ingeniería en Acuicultura y los grupos control por las carreras de Biología Marina e Ingeniería Ambiental.
Con el fin de lograr datos fidedignos de esta muestra, obtuvimos la confiabilidad y validez del instrumento de evaluación usado como pre- y posttest, a fin de determinar el grado de avance logrado durante un periodo de intervención pedagógica de 36 horas. También aplicamos dos instrumentos cualitativos, un cuestionario y un focus group, para complementar los resultados cuantitativos e indagar sobre las creencias, experiencias y conocimientos de los estudiantes sobre estrategias de comprensión de textos escritos, tanto en lengua materna (L1) como en la lengua extranjera (L2).

Con esta modalidad de análisis de textos disciplinares, buscamos responder al problema planteado anteriormente a través de la delimitación teórica de la interacción entre estrategias de procedimientos metodológicos provenientes de enfoques de enseñanza de lenguas y su operacionalización en lo que denominamos Marco Textual, que se basa exclusivamente en textos de la disciplina. Nuestro propósito fue obtener evidencia empírica sobre la efectividad de dicho modelo en el aprendizaje concerniente a la comprensión de textos académicos escritos en inglés.

\section{Marco teórico}

\section{Comprensión de textos escritos}

Al examinar algunas definiciones sobre la comprensión de textos escritos, encontramos que Jiménez (1998) la define como la relación entre elementos aparentemente disímiles en una sola totalidad, en una única estructura significativa ya que las diversas partes ocupan un orden dado de acuerdo con ciertos principios (esquemas, inferencias, etc.) que autorizan que los conceptos puedan ordenarse de una forma dada. León (2001, p.1) la entiende como "un proceso complejo e interactivo que requiere de la activación de una cantidad considerable de conocimiento por parte del lector y de la generación de un gran número de inferencias".

Parodi, Peronard e Ibañez (2010) afirman que para que un sujeto enfrente un texto escrito con éxito, es necesario que posea y active una serie 
de conocimientos de tipo lingüístico y contextual relacionados con el texto que lee. Por esta razón, esos conocimientos que son convencionalizados $\mathrm{y}$ estandarizados le permiten al lector distinguir y clasificar los textos en diferentes categorías más generales que se denominan géneros discursivos (Parodi et al., 2010).

Las definiciones anteriores se refieren a que comprender un texto escrito es un proceso complejo, interactivo que exige elaborar y aplicar conocimiento de manera coherente para relacionar, clasificar, ordenar, estructurar y construir conceptos de forma significativa.

Complementando lo anterior, Grabe y Stoller (2002) y Grabe (2009) postulan que hay dos tipos de procesos involucrados en la comprensión de lectura. Estos dos tipos son denominados procesos de nivel inferior y procesos de nivel superior. Entre los primeros tenemos el reconocimiento de palabras, la segmentación sintáctica (uso de información gramatical) y la codificación de las proposiciones semánticas (construcción del significado a nivel de cláusulas a partir de los significados de las palabras y de la información gramatical). Estos se llevan a cabo en la memoria operativa, la estructura en la cual se integran los procesamientos cognitivos y los recursos de conocimiento para la comprensión; sin estos procesos, la comprensión no puede ocurrir (Grabe and Stoller, 2002; Grabe, 2009). Los principales componentes de los procesos de nivel superior son los siguientes: un modelo de comprensión del texto, un modelo de situación interpretado por el lector y un número de recursos y habilidades de lectura regulado por el mecanismo de control ejecutivo de la memoria operativa (estrategias, metas, inferencias, conocimiento previo, monitoreo de la comprensión) (Grabe and Stoller, 2002; Grabe, 2009).

Para Koda (2005), el procesamiento consiste en una integración incremental significativa en unidades lingüísticas mayores al incorporar información sintáctica, semántica y pragmática. Ligado a este concepto está el de los mecanismos de conexión que implican no solo entender las funciones de los conectores, sino además una comprensión acabada de las cláusulas que deben ser conectadas.
Los propósitos de nuestra investigación nos obligan a comparar la lectura en la lengua materna (L1) y en la lengua extranjera (L2). Según Grabe y Stoller (2002), existen diferencias lingüísticas y de procesamiento entre ambas. En primer lugar, hay diferencias en la cantidad de conocimiento léxico, gramatical y discursivo en las etapas iniciales de la lectura en L1 y L2. Sin embargo, hay mayor conciencia metacognitiva y metalingüística en los contextos de L2. En segundo lugar, la cantidad de exposición a la lectura en L2 es diferente a la cantidad a la que están expuestos los sujetos en L1. Por último, hay que tomar en cuenta que existen diferencias lingüísticas entre las dos lenguas y diferentes competencias en L2 que sirven de base para la lectura en la lengua extranjera. Las influencias de transferencias entre las lenguas varían y también influye en la comprensión trabajar con dos lenguas distintas (Grabe and Stoller, 2002).

Además del procesamiento, otro factor importante son las estrategias que el lector utiliza cuando lee. Estas estrategias le permiten definir la tarea, buscar, localizar, sintetizar y evaluar información pertinente para identificar funciones textuales, implicaciones, relaciones, inferencias, etc., sobre todo si los textos están escritos en inglés, puesto que la falta de dominio de los sistemas léxico, gramatical y pragmático en L2 hace que los sujetos no transfieran a la lengua extranjera las estrategias aprendidas en la lengua materna (Koda, 2005).

Así, las estrategias reflejan el esfuerzo del estudiante para construir su propio conocimiento (Klassen, Lam, Chudley \& Forde, 2013). Ellas se entienden como la capacidad sistemática para transformar información en conocimiento, es decir, proceso y estrategia se implican mutuamente. Por esta razón, Oxford (1990) considera que las estrategias son operaciones empleadas por el aprendiz para facilitar la adquisición, almacenamiento, recuperación y uso de la información. Desearíamos ampliar esta definición indicando que la información debe tener el rango de conocimiento declarativo, procedimental y condicional que es lo que realmente se usa en las estrategias (Paris, Lipson and Wixon, 1994). Podríamos concluir diciendo que, a mayor conocimiento, la conceptualización es mayor lo que incrementa la capacidad para comprender y a su vez 
propicia mayor conocimiento, pudiendo transferirse a nuevas situaciones. De acuerdo con Gu (2005), el conocimiento humano progresa con la formación, delineación y refinamiento conceptual $\mathrm{y}$, por consiguiente, "con un mayor poder de generalización y explicación” (Oxford E Crookall, 1989, p. 415).

Por cuanto nuestra investigación se basa en textos disciplinares en inglés es necesario precisar qué se entiende por Inglés para Propósitos Académicos.

\section{Inglés para Propósitos Académicos}

Inglés para Propósitos Académicos (IPA) cubre todas las áreas de la práctica comunicativa académica, desde la enseñanza pre-terciaria, de pregrado y postgrado; géneros de investigación (artículos científicos, ponencias, conferencias, propuestas de investigación); escritura académica (ensayos, exámenes y tesis de grado), etc. (Swales, 1990). Específicamente, sus propósitos trascienden los contextos comunicativos e intentan entender la naturaleza del conocimiento disciplinario (Hyland, 2006), o sea, la adopción de nuevos roles, nuevos compromisos con el conocimiento, formas diversas de construir el conocimiento, todo esto dentro de una comunidad discursiva dada. Gracias al análisis del género, IPA puede interpretarse según el modo cómo los escritores usan el lenguaje para responder a situaciones recurrentes (Hyland, 2006). Es decir, es el estudio de la conducta lingüística situada en ambientes profesionales, académicos o institucionalizados. Así, para Hyland (2006), cada disciplina es una "tribu académica" con sus propias normas, nomenclaturas, estructuras de conocimiento y sus propias formas de investigar. Y dentro de cada cultura, los individuos adquieren competencias discursivas especializadas y necesarias para ser miembro del grupo, lo cual implica compartir conocimientos específicos y metodologías, especialmente formas de pensar, de construir y consumir el conocimiento, normas y epistemologías y, por sobre todo, las metas típicas y las prácticas de la disciplina para alcanzarlas (Bhatia, 2004).

Swales (1990) establece la relación existente entre una comunidad discursiva y las formas genéricas que produce, sugiriendo que los géneros pertenecen a las comunidades, no a los individuos. Del mismo modo, Bazerman (1988) establece una importante conexión entre la formación de una comunidad científica y la evolución de las estrategias discursivas que se utilizan para presentar reivindicaciones científicas en los artículos de investigación experimental.

Las últimas tendencias en el análisis del discurso científico-académico apuntan a que se deben examinar los tipos de textos y a los escritores en contextos y situaciones específicas, teniendo en cuenta factores como son el propósito y la audiencia. De este modo, la noción de "comunidad discursiva" ha adquirido gran relevancia recientemente, al asumirse que el discurso académico está supeditado a las convenciones establecidas por una comunidad determinada, ya sea científica o un grupo social (Martín, 2010).

Parodi et al. (2010) señalan la dificultad que enfrentan diversos lectores al leer textos escritos especializados, tanto de tipo académico como profesional. Tales géneros discursivos tienen una serie de características que pueden resultar desconocidas para los lectores y ello hace que su comprensión se dificulte. Es decir, dado que los sujetos que acceden a la universidad y al mundo laboral deben construir significados diferentes, estos géneros discursivos son nuevos no solo en su contenido temático sino también en su modo de organizar la información y en los propósitos que tienen.

Para contextualizar la investigación de los textos disciplinares escritos en inglés, Ibañez (2007) realizó un estudio científico-histórico en el cual distingue y compara conceptos fundamentales tales como los de comprensión y de lectura, tanto en lengua materna como en lengua extranjera, para delimitar investigaciones en esas áreas. Asimismo, los estudios acerca de la comprensión de textos disciplinares escritos en inglés y los estudios acerca de la comprensión tienen, como uno de sus principales puntos de convergencia, el concepto de comprensión y de aprendizaje a partir del texto. El autor concluye que la investigación acerca de la comprensión de textos disciplinares escritos en 
inglés no tiene como su objetivo central la enseñanza del inglés, sino el acceso a la disciplina a través del inglés y, en este sentido, esta línea de investigación surge como parte constituyente del estudio del IPA. Más aún, Ibañez (2007) propone que esta línea de investigación sea autónoma.

En otro artículo, Ibañez (2008) se centró en alumnos de Química Industrial $(n=84)$ y tuvo como objeto de estudio el proceso de comprensión logrado por estos estudiantes al enfrentar textos disciplinares escritos en inglés y cómo dicho nivel de comprensión es determinado por el nivel de dominio del inglés de los alumnos, su grado de inserción en la carrera y su habilidad para comprender textos en lengua materna. El estudio correlacional mostró que la relación entre el nivel de comprensión que los alumnos alcanzaron y cada una de las variables consideradas no representa una correlación positiva fuerte. La variable con mayor incidencia en el nivel de comprensión del texto académico escrito en inglés correspondió a la variable dominio del inglés, seguida de la variable inserción disciplinar $\mathrm{y}$, por último, a la variable habilidad de comprensión en lengua materna. Es interesante destacar que la variable dominio del inglés fue la de mayor incidencia debido al bajo logro obtenido al ser aplicado el instrumento Quick Placement Test, puesto que el 95\% de la muestra estudiada se distribuyó entre los niveles principiante a pre-intermedio.

\section{Hipótesis de interdependencia lingüística y del umbral lingüístico}

Según lo planteado anteriormente, es necesario comparar las diferencias y similitudes entre las competencias lectoras en L1 y L2. Inmediatamente planteado este problema surgen dos hipótesis para enfrentarlo.

La primera perspectiva -interdependencia lingüística - sostiene que las capacidades previas de los individuos en L1, afectan el desarrollo de las habilidades de lectura en L2. Por lo mismo, una escasa competencia lectora en L2 se debe a una escasa habilidad lectora en L1 o a estrategias incorrectas para leer en esa lengua extranjera, estrategias que difieren de aquellas para la lectura en la lengua materna (Koda, 2005).
Al contrario, la segunda hipótesis sostiene que una competencia lectora escasa se debe, ya sea a un conocimiento inadecuado de la L2 o a estrategias lectoras de L1 que no se utilizan en L2 por desconocimiento de L2. Un buen lector en L1 lo será en L2 cuando haya cruzado cierto umbral de habilidad lingüística en L2. La hipótesis del "umbral lingüístico" subraya la importancia del conocimiento de L2 como la fuente primordial de la inestabilidad en el desempeño.

En síntesis, la primera hipótesis argumenta que cualquier logro en L2 está determinado por la prioridad alcanzada en L1. Dado el tipo de comunicación que establece la lectura (descontextualizada), el fenómeno impone una complejidad cognitiva basada no solo en conocimiento lingüístico básico, sino además en una base cognitiva suficiente para manipular información que carece de estructuras referenciales de apoyo. Una vez logrado esto en L1, se puede esperar resultados positivos en L2. Sin embargo, se debe prestar atención a los siguientes hechos: es factible pensar que la conciencia metalingüística que es un buen predictor del éxito en competencia lectora en lectores novicios de L1, no lo es en aprendices adultos de L2 metalingüísticamente sofisticados; las habilidades conceptuales en L1 se hacen crecientemente significativas a medida que el contenido se hace más abstracto y complejo, lo que puede no darse en L2. Así entonces, no es factible afirmar una relación directa entre L1 y L2. En cambio, la segunda perspectiva privilegia la proficiencia en L2 como requisito para una comprensión eficiente de textos escritos en L2, puesto que un control limitado del sistema de L2 genera interferencias que transforman al sujeto en un lector que debe usar estrategias insuficientes y que pierde, por ejemplo, las habilidades de adivinar o predecir al desconocer las pistas correctas para hacerlo. En síntesis, “...un conocimiento limitado en L2, inhibe el uso de las habilidades previamente adquiridas en L1" (Koda, 2005, p. 23).

Para nuestro caso en particular, cabe destacar que parte de la dificultad en comprensión de lectura en L2 está dada por un conocimiento inadecuado de aspectos léxico-gramaticales y semánticos del inglés (morfología, composición y ordenamiento 
de la frase nominal inglesa, secuencia oracional, conectores, secuencias de oraciones, etc.). De aquí, entonces que sea necesario desarrollar este umbral lingüístico sobre todo en la gran mayoría de los alumnos que llegan a primer año de la universidad con muy poco conocimiento del inglés, como se explicó anteriormente.

\section{Metodología}

\section{Diseño de la investigación}

Este es un diseño cuasi-experimental con muestras naturalmente formadas por alumnos universitarios de primer año. Con respecto a la metodología de investigación, usamos metodología cuantitativa y cualitativa puesto que queríamos tener evidencias no solo del producto sino también del proceso de este estudio. De acuerdo con Sandelowski (2003) hay dos propósitos importantes para combinar métodos: (a) obtener una comprensión más completa de un fenómeno, mirándolo desde diferentes ángulos y (b) verificar una serie de hallazgos contrastándolos con otros datos; en otras palabras, validar nuestras conclusiones al presentar resultados convergentes obtenidos por medio de diferentes métodos.

\section{Sujetos}

Los sujetos fueron alumnos regulares de tres cursos de primer año de una universidad del sur de Chile. El grupo experimental estuvo formado por alumnos de la carrera de Ingeniería en Acuicultura $(n=42)$ y los dos grupos control por alumnos de la carrera de Biología Marina $(n=15)$ e Ingeniería Ambiental $(n=12)$. Tomamos dos cursos para el grupo control debido a una baja en el ingreso de alumnos en esas carreras durante el año que se realizó esta intervención.

\section{Procedimiento}

Los alumnos del grupo experimental fueron sometidos a una intervención pedagógica de 36 horas, en las cuales se aplicó una metodología de enseñanza basada en lo que denominamos
Marco Textual, que utilizó exclusivamente textos de la disciplina, usando estrategias de comprensión de textos escritos (identificación de información general y específica, identificar aspectos léxicos, gramaticales y semánticos, relacionar, clasificar, inferir y construir conceptos, resumir, etc.).

Por otra parte, los cursos de los dos grupos control, que también tuvieron 36 horas de clases, usaron un enfoque de enseñanza de lenguas más general, enfatizando el aprendizaje de vocabulario y gramática, pero estos contenidos no siempre estuvieron relacionados con textos de la especialidad.

\section{Objetivos}

Nuestros objetivos fueron: describir los factores que inciden en la complejidad de los textos de especialidad; determinar si la enseñanza basada en estrategias aumenta la comprensión de textos disciplinares escritos en inglés del ámbito de la Acuicultura y; por último, comparar y analizar las estrategias de comprensión de textos disciplinares escritos en inglés desarrolladas por los alumnos del grupo experimental y los grupos control después de la intervención pedagógica.

\section{Hipótesis}

HO: Las medias de los tres grupos son iguales.

H1: Las medias de los tres grupos no son todas iguales.

H0: Las medias del pre test y post test son iguales en los tres grupos.

H2: La media del pre test es menor que la del post test en los tres grupos.

El pre-test/post-test fue validado estadísticamente con 0,81 de confiabilidad por medio del coeficiente KR20 (Hernández, Fernández y Baptista, 2006) para determinar el grado de avance logrado durante un periodo de intervención pedagógica. Este instrumento (pre y post test) intentaba medir lo siguiente: 
Ítem 1. Información General:

Identifica datos de cobertura amplia: los conceptos sobre los que se comentan diversos aspectos.

\section{Ítem 2 y 3. Información específica:}

Identifica aspectos particulares, puntuales de conceptos, relaciones, etc., sin precisar relaciones con el resto del texto.

\section{Ítem 4. Lexis:}

Manejo de significado léxico, relaciones léxicas y connotaciones de las palabras.

\section{Ítem 5. Significado sintáctico:}

Significado, relaciones intra, inter oracionales, funciones (propósito, causa efecto, condición, etc.).

\section{Ítem 6. Semántica léxica:}

Rasgos léxicos y relación en un contexto (oración).

Ítem 7.

A. Relación concepto(todo)-partes:

Identifica un concepto y sus partes, atributos, posición, función en el texto, etc.

B. Información específica:

Referida a las relaciones particulares en un párrafo dado.

\section{Relación causa-efecto:}

Establece la relación correcta entre causa y efecto o viceversa.

Los instrumentos cualitativos incluyeron un cuestionario para indagar acerca del conocimiento de los estudiantes sobre estrategias de comprensión de textos escritos y un focus group. Los textos usados en la intervención pedagógica fueron artículos científicos tomados de revistas de la especialidad de Acuicultura. El material para la intervención pedagógica se estructuró utilizando actividades cognitivas o estratégicas de modo que, al solucionar los problemas allí planteados, los alumnos pudieran aplicar y desarrollar conocimiento léxico, sintáctico y habilidades discursivas específicas; además de estrategias de comprensión de textos escritos.

\section{Resultados}

El porcentaje de logro alcanzado por los alumnos del grupo experimental y los grupos control en el pre-test fue de $30,1 \%$, de $28,4 \% \%$ y $34,6 \%$, respectivamente. Es decir, demostraron un umbral lingüístico no aceptable para leer comprensivamente en inglés, en cuanto a conocimiento léxico, sintáctico y relaciones intra texto. El conocimiento de la L2 con que llega la gran mayoría de los alumnos a la universidad en Chile es escaso y coincide con los resultados descritos en los estudios de Ibañez (2008), Arancibia (2010) y Lagos (2011).

El asunto entonces era cómo ayudarlos a transformarse en lectores competentes. Al analizar los textos de la especialidad descubrimos características recurrentes que pueden ayudar a los alumnos a inferir significados en forma rápida. Hay características, relaciones y aspectos de este género discursivo que pudimos detectar y que son bastante fáciles de encontrar en los textos que deben leer los alumnos de Acuicultura; nos referimos a las unidades y relaciones.

Para facilitar la transformación de nuestros alumnos en lectores competentes, necesitábamos construir actividades que incluyeran los conceptos discutidos hasta aquí. Todo este constructo lo denominamos Marco Textual y se explica a continuación.

\section{Concepto de Marco Textual}

Partiendo del concepto de géneroy considerando los conceptos ya discutidos, elaboramos un componente que facilita la comprensión, es lo que hemos denominado Marco Textual. En principio, 
todo texto se refiere a un mundo vivo, dinámico, constituido por entes (con características, atributos y comportamientos propios) capaces de establecer relaciones (jerarquías, órdenes, estructuras etc.) para mantener y proyectar los sistemas que los sustentan. Según nuestros análisis cada uno de estos mundos es una mirada distinta, para distinguir entes y relaciones de diversa magnitud. Lo interesante de esta perspectiva es que es una visión sintética de un dominio de conocimiento y a la vez integradora de la indagación de la realidad; es un nivel que muestra la forma cómo un dominio de conocimiento usa el pensamiento para elaborar esa realidad y la gramática para elaborar su propio discurso.

En un análisis a nivel léxico, gracias al Marco Textual, podemos ver cómo un acceso léxico ordena y organiza los contenidos en un texto y facilita la comprensión de ciertas unidades. En este caso, plaga e infecciones, y el tipo de relaciones en que ellas se encuentran:

1. xplague is causedby the parasite saprolegmious.

2. The parasite is endemic to [place].

3. The parasite is specialized to penetrate into [place]

4. The species are able to limit an infection.

5. Immune response is possible due to produce prophenoloxidase.

6. In North American species, the parasite survives despite being inhibited in growth, and

7. Its infective units- zoospores- may be released into the water

8. Infected American crayfish serve as vectors of the disease.

9. Crayfish plague was spread into [place]at the end of the 1850s, and

10. During the end of the $19^{\text {th }}$ century it eradicated a huge number of European crayfish across the whole continent.

11. Crayfish mass mortality caused by a. Astaci has been recently recorded in [places].

Nótese que el léxico a estudiar no responde a una memorización de palabras aisladas sino que se privilegia el orden jerárquico o lógico propio de este texto.
Asimismo, si al considerar las oraciones, nos percatamos de la relación lógica existente entre el concepto "plague" y "cause, spread, eradicate, record, release", etc., se hace notoria, además, una estructura preponderante de voz pasiva. Se integra así un tipo de léxico determinado a una estructura dada y a una relación específica de descripción y causalidad. Gradualmente, además, se va ingresando el concepto de longitud de oración que constituye una dificultad básica en la comprensión de lectura.

El Marco Textual es efectivo en referencia al conocimiento, competencia o conocimiento de la lengua y al desempeño que un sujeto tiene en un dominio dado para explicar, crear y desarrollar esa realidad en el ámbito real o imaginario de una actividad. Este conocimiento que le permite enfrentarse a la lectura de un texto, responde a un criterio cognitivo de organización de relaciones en un grado altamente abstracto y sintético. Establece una cierta carta de procedimiento y de categorías relacionales propias de un dominio con posibilidades de combinación ilimitada. Se convierte así en una base de conocimiento general típica. Lo importante de este Marco Textual es que tiene la capacidad de integrar elementos discursivos con elementos lingüísticos a la vez que permite al lector manejar conjuntamente los componentes anteriores y el género académico respectivo.

En general, se puede afirmar que todo texto que pertenece a un área de conocimiento dado organiza la realidad según sus propios intereses, principios, objeto y modo de análisis. Vale decir, una dificultad inmediata que esta investigación debe solucionar es el tipo de conocimiento, ya sea de intereses, principios, objeto y modo de análisis, que distingue a este texto de otros textos del mismo nivel. De esta manera, para comprender un texto del dominio de la Acuicultura, es necesario considerar una construcción determinada por las categorías de análisis incluidas en la tabla 1.

Al ser el concepto Marco Textual genérico para todo texto de acuicultura en artículos de revistas especializadas, permitió enfocar el texto de un modo más organizado y en trozos mayores con una 
Tabla 1. Dominio de las ciencias (Acuicultura)

\begin{tabular}{|c|c|}
\hline Unidades & \\
\hline Entidades & $\begin{array}{l}\text { Diversidad de sujetos/objetos (especies): características, hábitat, } \\
\text { generación, producción, manipulaciones (intervenciones); sobrevivencia, mortandad, } \\
\text { hereditabilidad, etc. }\end{array}$ \\
\hline Cantidades & De entidades, de elementos necesarios para dichas entidades, dinero invertido, generado, etc. \\
\hline Tiempo & Necesario para intervenir, aumentar entidades y cantidades. \\
\hline Mejoras & $\begin{array}{l}\text { Producidas por las intervenciones, riesgos, costos, peligros (enfermedades y su control). Selección } \\
\text { del mejor ítem (peso, edad). }\end{array}$ \\
\hline Mediciones & $\begin{array}{l}\text { Probablemente lo más importante de esta construcción, deben realizarse para justificar los puntos } \\
\text { anteriores. Selección del mejor ítem (peso, edad). }\end{array}$ \\
\hline Fines & Pragmático: económico-industrial; gestar ganancias. Selección del mejor ítem(peso, edad). \\
\hline $\begin{array}{l}\text { Métodos, } \\
\text { Estudios }\end{array}$ & Uso de métodos conocidos, identificación de problema, impactos, antecedentes. \\
\hline Enfermedades & Tratamiento, costo, dosis. \\
\hline \multicolumn{2}{|l|}{ Relaciones } \\
\hline Descripción & Especies, objetos, dimensiones. \\
\hline Control & Ejercido en una determinada entidad, lugar o tiempo dado. \\
\hline Causa-efecto & $\begin{array}{l}\text { Antecedente-consecuente, proporción, razón, correlación, etc., } \\
\text { de aquí, lo determinante de la intervención y los fines. }\end{array}$ \\
\hline Proceso & Requisitos, procedimientos, etapas, programas. \\
\hline Comparación & De rasgos, de calidades (peso, edad, crecimiento), de hábitats, etc. \\
\hline Selección & Rasgos, condiciones, variaciones (peso, edad) que determinan la selección \\
\hline Evaluación & Variables, resultados, planteamientos estadísticos. \\
\hline Jerarquías & Interacción, competencia, dominación, estabilidad, división. \\
\hline
\end{tabular}

mayor incidencia en la capacidad de procesamiento del sujeto al poder relacionar una mayor cantidad de información y de organizar, relacionar e integrar los hechos.

En el texto (Kozubíková et al., 2008)

Two-year-old signal crayfish $P$ leniusculus juveniles were reared communally in plastic tanks $(0.8 \mathrm{~m} \times 0.6 \mathrm{~m} \times 0.3 \mathrm{~m}$, water depth 0.15 $\mathrm{m}$, area $0.48 \mathrm{~m} 2)$ in a partially $(90 \%)$ recirculating

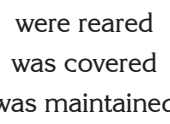

water system. The tank bottom was covered with a 3-4 cm thick layer of mixed gravel (1015 $\mathrm{mm}$ in diameter) and limestone (5-10 mm). Each tank had its own water inlet (inflow $1.51 \mathrm{~min}$ -1) and outlet. Temperature of the aerated and filtered groundwater was maintained at $20^{\circ} \mathrm{C}$ with a thermostatically-controlled heater. (p. 2)

escogido para ejemplificar la Unidad "especie" (crayfish) y la Relación "descripción", notamos el siguiente ordenamiento
Se hace notorio que la Descripción considere elementos cuantitativos como " $3-4 \mathrm{~cm}$ ", "20C" y que ello ocurra EN lugares especiales, A ciertas temperaturas que se logran CON ciertos instrumentos (calefactor) o se cubren CON algo (una capa) DE una constitución determinada. Al 
respecto, cabe señalar tres aspectos: el primero es que la descripción opera exactamente como lo muestra este texto, es decir, entrega rasgos de los elementos importantes que se relacionan con el tema del texto (crayfish). Lo segundo, es que la descripción usa pocos conectores, salvo el punto seguido ya que la relación de estos objetos es solo de contigüidad. Finalmente, la construcción gramatical es simple: oraciones cortas y simples marcadas por la cópula "Ser-Estar", típica de esta relación. La tradicional voz pasiva pasa a ser un elemento más en este tipo de relación y no constituye dificultad alguna para la comprensión. Existe sin embargo un hecho de mayor relevancia: éste es la relación lógico semántica que se logra con la comprensión de las frases verbales de este texto (relaciones intra oracionales) como lo indicamos al inicio de este párrafo, o sea, implicaciones, inferencias, etc. También importa destacar que el Marco Textual facilita la identificación de colocaciones: "plastic tanks, water depth, recirculating water system", etc.

Otras "Relaciones" para otras "Unidades" se conforman de manera distinta por ser los textos altamente dinámicos y responder a otras necesidades explicativas. Asimismo, al comparar este Marco Textual con aquél de otra disciplina se notará que tanto las Unidades como las Relaciones cambian. Estos cambios también se manifiestan en los niveles léxico, sintáctico y semántico. Sin embargo, dicha diversidad está acotada y es factible organizarla de modos eficientes para efectos de un aprendizaje exitoso.

En términos más específicos, otro nivel de dificultad que aumenta la complejidad de este tipo de discurso es el manejo de los siguientes aspectos lingüístico-discursivos:

- Estructura textual: género, funciones, actos de habla, marcadores discursivos.

- Componentes oracionales: Frases nominales, frases verbales y la secuencia tipo del sistema lingüístico del Inglés. [NP $\left.{ }^{1}+\mathrm{VP}+\left(\mathrm{NP}^{2}\right)+\mathrm{K}\right]$. Las funciones de estas frases [Actor/agente $\left(\mathrm{NP}^{1}\right)$; Meta, beneficiario (NP2 $)$, etc.].

- Segmentación (parsing) de oraciones: longitud de la oración (conector).

- Conectores y sus funciones: Conceptos, atributos y relaciones

- Nivel léxico: colocaciones, sustantivos compuestos, preposiciones, funciones y sentido de las preposiciones.

\section{Resultados instrumentos cuantitativos}

Para comparar si las muestras provenían de distribuciones normales, se aplicó el sesgo estandarizado y la curtosis estandarizada a los tres grupos. Los valores de estos estadísticos indicaron que los tres grupos se encuentran dentro del rango esperado.

\section{Análisis del pre/post-test del grupo experimental}

El logro promedio en el pre-test de los alumnos del grupo experimental fue de $30,1 \%$; sin embargo, este subió a $45,87 \%$ en el post-test.

Con respecto al pre-test y post-test, los niveles de logro en el grupo experimental, expresados en rangos de porcentajes, se encuentran en la tabla 2.

Tabla 2. Porcentajes de logro del grupo experimental: Ingeniería en Acuicultura

\begin{tabular}{cccccc}
\hline $\begin{array}{c}\text { Rangos de porcentajes } \\
\text { de logros }\end{array}$ & \multicolumn{2}{c}{$\begin{array}{c}\text { Número de alumnos } \\
\text { por rango }\end{array}$} & \multicolumn{2}{c}{$\begin{array}{c}\text { Porcentaje respecto } \\
\text { del total de alumnos }\end{array}$} \\
\hline Pre-test & Post-test & Pre-test & Post-test & Pre-test & Post-test \\
\hline $0 \%-29 \%$ & 17 & 1 & $40,47 \%$ & $2,38 \%$ \\
$30 \%-39 \%$ & 14 & 5 & $33,33 \%$ & $11,90 \%$ \\
$40 \%-49 \%$ & 8 & 27 & $19,04 \%$ & $64,28 \%$ \\
$50 \%-59 \%$ & 3 & 6 & $7,14 \%$ & $14,28 \%$ \\
$60 \%-79 \%$ & 0 & 3 & $0 \%$ & $7,14 \%$ \\
$80 \%-100 \%$ & 0 & 0 & $0 \%$ & $0 \%$ \\
\hline
\end{tabular}


Análisis del pre/post-test de los grupos control

Respecto del pre-test y post-test, los niveles de logro en los grupos control, expresados en rangos de porcentajes, se encuentran en las tablas 3 y 4 .

Biología Marina subió de 28,4\% de logro en el pre-test a $37,6 \%$ en el post test e Ingeniería Ambiental de 34,6\% en el pre test bajó a 33,9\% en el post test.

Para analizar la relación entre los tres grupos, se utilizó la prueba estadística ANOVA (tabla 5). En el pre test, se comprobó que el valor-P $(0,6834)$ de la razón-F es mayor o igual que 0,05, o sea, no existe una diferencia estadísticamente significativa entre las medias de las tres variables con un nivel del 95,0\% de confianza. Se rechaza la hipótesis alternativa $\left(\mathrm{H}_{1}\right)$ y se verifica la hipótesis nula $\left(\mathrm{H}_{0}\right)$.

Por el contrario, la prueba estadística ANOVA, aplicada después del pos test, mostró que el valor-P $(0,0031)$ de la prueba-F es menor que 0,05 , o sea existe una diferencia estadísticamente significativa entre las medias de las 3 variables con un nivel del 95,0\% de confianza (tabla 6). Se rechaza la hipótesis nula $\left(\mathrm{H}_{0}\right)$ y se verifica la hipótesis alternativa $\left(\mathrm{H}_{1}\right)$.

Tabla 3. Porcentajes de logro del grupo control: Biología Marina

\begin{tabular}{|c|c|c|c|c|}
\hline Rangos de porcentajes de logros & \multicolumn{2}{|c|}{ Número de alumnos por rango } & \multicolumn{2}{|c|}{ Porcentaje respecto del total de alumnos } \\
\hline Post-test & Pre-test & Post-test & Pre-test & Post-test \\
\hline $0 \%-29 \%$ & 9 & 6 & $36,0 \%$ & $24,0 \%$ \\
\hline $30 \%-39 \%$ & 7 & 7 & $28,0 \%$ & $28,0 \%$ \\
\hline $40 \%-49 \%$ & 5 & 7 & $20,0 \%$ & $28,0 \%$ \\
\hline $50 \%-59 \%$ & 4 & 4 & $16,0 \%$ & $16,0 \%$ \\
\hline $60 \%-79 \%$ & 0 & 1 & $0 \%$ & $4,0 \%$ \\
\hline $80 \%-100 \%$ & 0 & 0 & $0 \%$ & $0 \%$ \\
\hline
\end{tabular}

Tabla 4. Porcentajes de logro del grupo control: Ingeniería Ambiental

\begin{tabular}{|c|c|c|c|c|}
\hline Rangos de porcentajes de logros & \multicolumn{2}{|c|}{ Número de alumnos por rango } & \multicolumn{2}{|c|}{ Porcentaje respecto del total de alumnos } \\
\hline Post-test & Pre-test & Post-test & Pre-test & Post-test \\
\hline $0 \%-29 \%$ & 5 & 5 & $36,0 \%$ & $24,0 \%$ \\
\hline $30 \%-39 \%$ & 1 & 1 & $28,0 \%$ & $28,0 \%$ \\
\hline $40 \%-49 \%$ & 4 & 3 & $20,0 \%$ & $28,0 \%$ \\
\hline $50 \%-59 \%$ & 2 & 1 & $16,0 \%$ & $16,0 \%$ \\
\hline $60 \%-79 \%$ & 0 & 0 & $0 \%$ & $0 \%$ \\
\hline $80 \%-100 \%$ & 0 & 0 & $0 \%$ & $0 \%$ \\
\hline
\end{tabular}

Tabla 5. ANOVA Pre test

\begin{tabular}{cccccc}
\hline Fuente & Suma de Cuadrados & GI & Cuadrado Medio & Razón-F & Valor-P \\
\hline Entre grupos & 131,326 & 2 & 65,663 & 0,38 & 0,6834 \\
Intra grupos & 10630,1 & 62 & 171,454 & & \\
Total & 10761,4 & 64 & & & \\
\hline
\end{tabular}

Tabla 6. ANOVA Post test

\begin{tabular}{cccccc}
\hline Fuente & Suma de Cuadrados & GI & Cuadrado Medio & Razón-F & Valor-P \\
\hline Entre grupos & 931,156 & 2 & 465,578 & 6,33 & 0,0031 \\
Intra grupos & 4779,71 & 65 & 73,534 & & \\
Total & 5710,87 & 67 & & & \\
\hline
\end{tabular}


Análisis por ítems entre pret test y post test grupo experimental Acuicultura y grupos control de Biología Marina e Ingeniería Ambiental

Se compararon las medias por ítem entre el pre test y el post test, con intervalos de confianza del 95\%. Se usó la prueba t para comparar los ítems de ambas pruebas. Los resultados son los siguientes: el grupo experimental (Acuicultura) obtuvo en todos los ítems un valor-P menor que 0,05. El grupo control de Biología Marina, solo en el primer ítem obtuvo un valor-P menor que 0,05 , en el resto de los ítems, el valor-P obtenido fue mayor a 0,05. En el grupo control de Ingeniería Ambiental, el valor-P obtenido en todos los ítems fue mayor a 0,05.

El avance del grupo experimental se grafica en la figura 1.

Este gráfico permite visualizar los siguientes aspectos:

1. Muestra claramente la zona de desarrollo próximo para cada alumno. Se comparan los puntajes tabulados individuales para cada ítem de los alumnos.
2. La tendencia descendente en rojo (pre test) se revierte y se hace más lineal en azul (post test), se puede observar el avance de los estudiantes.

3. Se observa que quienes más avanzan son los que sabían menos al inicio del proceso.

4. Los efectos positivos residen en los resultados de las estrategias de comprensión de textos escritos basadas en textos disciplinares que permiten efectuar cambios en el aprendizaje y lograr una imagen precisa de las habilidades de cada sujeto en particular y del grupo en general.

\section{Análisis de los instrumentos cualitativos}

\section{Encuesta}

Los instrumentos cualitativos aplicados fueron un cuestionario y una entrevista focalizada (focus group).

La característica esencial de una encuesta es su empleo de categorías y sus rasgos pueden ser precisamente definidos por los investigadores de antemano (Dörnyei, 2003).

La encuesta tenía como propósito indagar sobre el conocimiento metacognitivo de los

Figura 1. Resultado pre y post-test

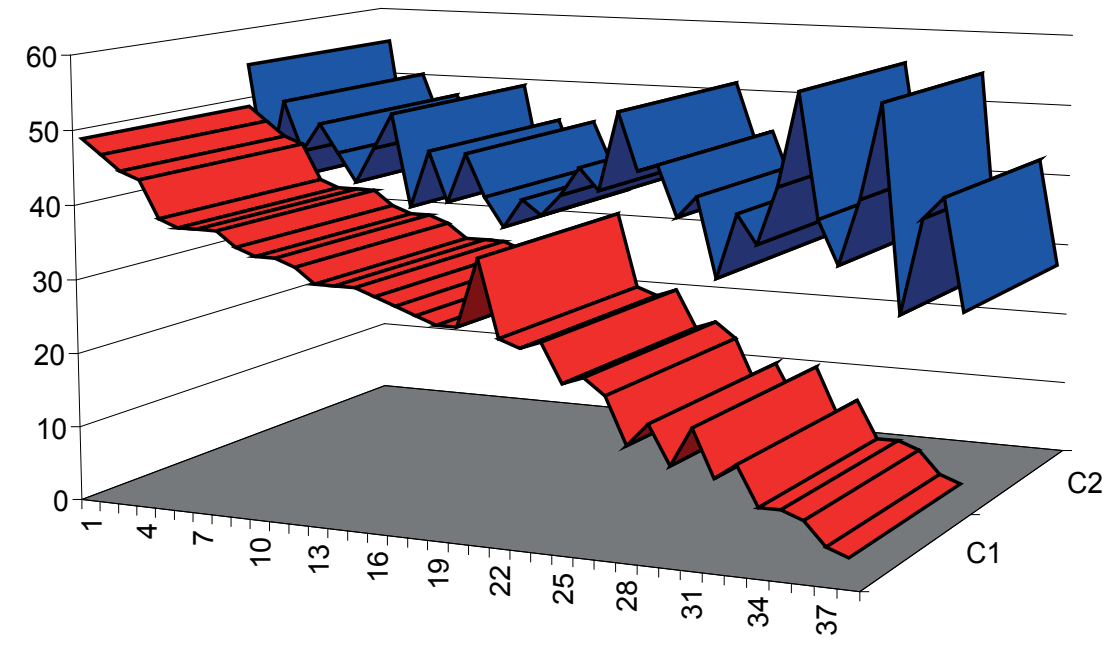

Fuente: Elaboración propia. 
alumnos universitarios acerca de la comprensión de textos escritos. Como ya se sabe, el conocimiento metacognitivo incluye los subprocesos conocimiento declarativo (conocimiento sobre hechos, sucesos, lenguaje, conceptos y teorías acerca del mundo), conocimiento procedimental (el repertorio de acciones que el lector aplica a la tarea) y conocimiento condicional (uso de estrategias, la comprensión del contexto social y el propósito del lector) (Paris, Lipson y Wixson, 1994) (tabla 7).

La encuesta estaba dividida en cuatro categorías y dos subcategorías de acuerdo con la tabla 7. Por medio de este instrumento, solo tratamos de evaluar el conocimiento declarativo y el procedimental.

La encuesta contestada por el grupo experimental al empezar el período de intervención mostró que los alumnos no estaban conscientes de las estrategias que manejaban.

En la sección de Planificación (56,76\%), la mayoría indicó que le daba una mirada general al texto y podían obtener la idea general por medio del título y subtítulos.

En la sección de Monitoreo, seis alumnos obtuvieron 0 punto. Este hecho se podría explicar por las dificultades encontradas al procesar la información mientras van leyendo. Estas les impiden identificar el tópico del texto, resumirlo y también dificulta la identificación de relaciones (causa/efecto, condición, propósito, etc.) dentro del texto. El porcentaje obtenido fue de $29 \%$ : lo cual sugiere que muy pocos alumnos monitoreaban su comprensión mientras leían.
En la sección evaluación, el porcentaje alcanzado fue de $47,8 \%$, por lo tanto, menos del $50 \%$ puede determinar si comprendieron o no, si identifican los principios de organización del texto y cuál es la información importante.

Con respecto a la cuarta sección, el conocimiento metacognitivo, también arrojó bajos resultados: $41 \%$. Detectamos problemas para identificar las colocaciones, las palabras que relacionan los párrafos, y el propósito del texto. Al encontrar una parte difícil, los estudiantes indicaron que solo releían tratando de adivinar el significado de las palabras desconocidas por el contexto; también, averiguamos que tenían dificultades para identificar los párrafos complejos en un texto y las causas o fuentes que generaban las dificultades.

\section{Focus group}

Para triangular la información recogida en el pretest y en la encuesta, realizamos un focus group con una muestra de ocho alumnos de Ingeniería en Acuicultura elegidos al azar. La información obtenida fue bastante útil ya que nos ayudó a entender los procesos que realizan los alumnos al tratar de comprender un texto y las creencias construidas durante sus años de permanencia en la educación básica y media con respecto a la comprensión de textos escritos en lengua materna y en lengua extranjera.

Es bastante difícil establecer una definición simple para el concepto de creencia debido a que diferentes autores lo entienden desde una perspectiva personal. Algunos autores se refieren

Tabla 7. Encuesta

\begin{tabular}{cc}
\hline Categoría & Subcategorías \\
\hline Planificación & \\
Monitoreo & \\
Evaluación & Conciencia acerca de la estructura textual \\
Conocimiento meta-cognitivo & Estrategias remediales \\
\hline
\end{tabular}


a las creencias como cognición, conocimiento, concepciones de enseñanza, conocimiento pedagógico, conocimiento práctico, teorías prácticas, orientaciones teóricas, actitudes, percepciones, etc. (Borg, 2006). En lo que sí hay acuerdo es que las creencias están profundamente enraizadas y son resistentes al cambio (Richards, Gallo E Renandya, 2001). Diferentes estudios han comprobado que las experiencias prácticas de los profesores pueden influir en sus creencias de diferentes maneras y que sus creencias influyen en las estrategias instruccionales que eligen las que, a su vez, impactan en la calidad del aprendizaje de los estudiantes (McKenzie, 1996; Schuh, Walker, Kizzie and Mohammed, 2001; Entwistle, McCune E Hounsell, 2002). Por otra parte, las creencias de los estudiantes tienen impacto en sus propias prácticas de aprendizaje (Taylor, 1996; Kember \& Wong, 2000; Johnston, 2001).

Las preguntas del focus group fueron dirigidas a averiguar qué tipo de libros, materiales, artículos, etc. leían; si se consideraban buenos o malos lectores; en qué consistía la complejidad de ciertos textos; y si era más difícil leer en inglés que en español. Los sujetos contestaron que leían esencialmente libros y guías de su especialidad, eran muy pocos los que leían novelas u otro tipo de género discursivo. Algunos leían el diario, generalmente en internet. Los estudiantes afirmaron que uno de los aspectos que dificultaba su comprensión en los artículos y libros que leían era el vocabulario desconocido o la falta de vocabulario, y el escaso conocimiento sobre el tópico. Respecto de si eran buenos o malos lectores contestaron

Yo me considero un mal lector porque entiendo muy poco de lo que leo, o a veces leo y me acuerdo de pocas cosas y tengo que volver a leer y por eso pienso que soy un mal lector y un mal comprendedor.

\section{Otro afirmó}

Yo me considero un mal lector en el sentido que hay tantos libros, tantas cosas interesantes que uno pudiera leer, pero no lo hago, a pesar de que cuando leo algo, lo entiendo, pero no me llama mucho la atención leer.

\section{En cambio, una alumna señaló}

Yo no me considero ni buena ni mala lectora, porque si fuera mala, no comprendería lo que leo y no tengo problemas en ese sentido. Ahora si fuera una excelente lectora, leería todos los días y no lo hago. Cuando tengo que leer, leo, y cuando encuentro algo de mi interés también.

Cuando tenían dificultades para entender un texto en la lengua materna, usaban otras estrategias, tales como hacer resúmenes, buscar la misma información en otras fuentes y también aclaraban ciertas ideas que no entendían con compañeros de niveles superiores.

Leo varias veces hasta entenderlo o hasta que me lo aprenda, o si no intento otros métodos como buscar resúmenes o buscar información de lo que vamos a leer; también le pregunto a compañeros de niveles superiores; los resúmenes ayudan porque entregan como una guía para poder llegar a una conclusión.

Es decir, estos alumnos no estaban conscientes sobre los procesos cognitivos que se ponen en juego para leer textos escritos y no distinguían entre "comprender", "memorizar" y "aprender". No bastaleer varias veces para entender un texto, la dificultad radica en "construir conceptos cada vez más abstractos y, al mismo tiempo, más específicos" (Parodi et al., 2010, p.32). La dificultad para distinguir que leer repetidas veces no es sinónimo de comprensión, significa que no hay desarrollo de las competencias que posee un buen lector. Según Neira, Reyes y Riffo (2015), la eficacia de los lectores hábiles radica en usar un mayor número de estrategias de comprensión lectora lo que, a su vez, conlleva una mayor reflexión.

Con respecto a los textos en inglés, una creencia muy arraigada es que al traducir un texto aprenden vocabulario y lo pueden entender. La mayoría cree que saber vocabulario ayuda a la comprensión "yo creo que lo que nos ayuda es el vocabulario, porque haciendo el trabajo uno va aprendiendo las palabras"; "en inglés a veces uno no comprende nada, en castellano uno a veces entiende". Un alumno señaló que "las palabras parecidas al castellano (cognados) me ayudan". 
Además, "la mayoría de las clases de comprensión lectora en inglés se reducía a traducir un texto". Estas aseveraciones corroboran los resultados de un estudio de Díaz, Martínez, Roa y Sanhueza (2010), que analiza las cogniciones pedagógicas de diez profesores de inglés del sistema educacional público de Chile en el nivel de enseñanza secundaria. Entre los modelos didácticos predominantes en sus discursos se encuentran: Gramática-traducción, Audio-lingual y Comunicativo.

Otra causa de la mala preparación que traen estos alumnos se lo atribuyen al cambio de profesores y a que todos los años los profesores les enseñaban los mismos contenidos (gramática)

A mí de primero a segundo me cambiaron mucho de profesor, y uno tenía un método de enseñar y otros, otro método. $Y$ nos hacían traducir y después nos daban un resumen...; pero es que igual van enseñando lo mismo todos los años; todos los años las mismas formas de los verbos; a mí lo que más me enseñaron eran frases y las fórmulas para hacer las frases.

En este aspecto, hay coincidencia con los hallazgos de Barahona (2014) quien, al examinar las experiencias previas de profesores en formación, averiguó que la mayoría de los profesores en la enseñanza secundaria, según los informantes, enfatizaban la enseñanza de la gramática, les hacían memorizar reglas gramaticales y les daban guías a los estudiantes con ejercicios para completar las formas de los verbos.

Al pedir sus opiniones sobre el periodo de intervención pedagógica con el Marco Textual, estas fueron bastante positivas:

La clase era interesante y entretenida; era más dinámica para aprender; yo creo que el método que están implementando es interesante. Las falencias que teníamos que eran las preposiciones, no me las sé de memoria, pero hice una lista, entonces sé dónde empieza y termina la frase ahora. Cuando uno trabaja en grupos con el profesor, está más en confianza y uno se atreve a preguntar las dudas. Es difícil preguntar frente a todo el curso.
Los aspectos interesantes para los alumnos fueron que el vocabulario y la gramática no se enseñaban aislados sino en el contexto de los textos de su disciplina. Y, además, las oraciones no se enseñaban aisladas sino insertas en el contexto de un discurso relacionado con los tópicos de su especialidad (Marco Textual). Es decir, fueron capaces de desarrollar una comprensión integral para dar sentido a las palabras, las frases y las oraciones en el contexto de sus textos disciplinares.

\section{Conclusiones}

Nuestros objetivos fueron describir los factores que inciden en la complejidad de los textos de especialidad; determinar si la enseñanza basada en estrategias aumenta la comprensión de textos disciplinares escritos en inglés del ámbito de la Acuicultura y, finalmente, comparar y analizar las estrategias de comprensión de textos disciplinares escritos en inglés desarrolladas por los alumnos del grupo experimental y los grupos control después de la intervención pedagógica.

A través del análisis de los artículos científicos de la especialidad pudimos construir ciertas categorías de análisis como unidades y relaciones que pertenecen exclusivamente al ámbito de la Acuicultura y que se denominó Marco Textual. Con las características, relaciones y aspectos de este género discursivo construimos las actividades y los instrumentos de evaluación para la intervención pedagógica llevada a cabo con el grupo experimental.

Al comparar los resultados del pre test en los tres grupos, encontramos que el porcentaje de logro y las medias fueron muy bajos; lo que coincide con los resultados encontrados en las investigaciones de Ibañez (2008); Arancibia (2010) y en los resultados del SIMCE inglés 2010, 2012 y 2014 (Agencia de la Calidad, 2013, 2015). La comprensión lectora en L2 de los tres grupos adolecía del conocimiento adecuado de aspectos léxico-gramaticales y semánticos del inglés y de competencias discursivas para relacionar, clasificar, inferir y construir conceptos de forma significativa. 
Una de las estrategias menos logradas en el pre-test en los tres grupos fue la referida a las relaciones (causa-efecto, propósito, comparacióncontraste, etc.). Este aspecto nos llama la atención por cuanto estos contenidos están considerados en los programas de estudio de la asignatura Lenguaje y Comunicación de Educación Media (Mineduc, 2009).

Respecto del grupo experimental, los resultados de la encuesta y el focus group mostraron que las estrategias de comprensión de textos escritos que manejaban estos alumnos en su lengua materna eran mínimas, por lo tanto, no podían transferirlas a la lengua extranjera y, además, no tenían el umbral lingüístico requerido (Koda, 2005), según los resultados del pre-test, para poder entender un texto disciplinar en inglés. Los géneros discursivos disciplinares tienen una serie de características desconocidas para los alumnos lo que dificulta su cabal comprensión. Así, los resultados obtenidos en el pre-test, en la encuesta y en el focus group demostraron no solo falta de estrategias de comprensión de textos escritos sino también de habilidades para establecer relaciones, interpretar e inferir (Mineduc, 2009). En el focus group, los alumnos mencionaron que en las clases de inglés durante la Enseñanza Media solo les enseñaban gramática y los textos de lectura se traducían; por esta razón, no habían desarrollado estrategas de comprensión lectora. Por lo tanto, fue necesario desarrollar más conocimiento de la L2 y de las características de los textos disciplinares de Acuicultura en el grupo experimental debido a este escaso conocimiento. Sin embargo, al poder distinguir con claridad cuáles eran las características del discurso de su especialidad después del período de intervención, los sujetos del grupo experimental tuvieron la posibilidad de acceder al conocimiento disciplinar, al comprender no solo el contenido temático sino también el modo de organización de la información y los propósitos que persiguen estos textos disciplinares.

Por ende, la aplicación del post-test al grupo experimental evidenció que la metodología aplicada con los alumnos de Acuicultura, basada en el Marco Textual y en el desarrollo de estrategias de comprensión, había sido efectiva. Según la comparación de medias, la ganancia obtenida por el grupo experimental entre el pre y el post test fue bastante amplia, hubo 13,62 puntos de diferencia a favor de los estudiantes de Acuicultura al comparar ambas pruebas. En cambio, la ganancia de los otros dos grupos fue menor: Biología Marina tuvo una ganancia de 9,15 puntos e Ingeniería Ambiental bajó de 30,9 a 30,2, es decir, obtuvo -0,7 de ganancia.

En los resultados por ítems, el grupo experimental (Acuicultura) obtuvo en todos ellos un valor-P menor que 0,05. El grupo control de Biología Marina, solo en el primer ítem obtuvo un valor-P menor que 0,05 , en el resto de los ítems, el valor-P obtenido fue mayor a 0,05. En el grupo control de Ingeniería Ambiental, el valor-P obtenido en todos los ítems fue mayor a 0,05. Esto significa que en el grupo experimental hubo incremento en todos los ítems que medían estrategias, sobre todo en los relacionados con la identificación de información específica y con el manejo del significado léxico, relaciones léxicas y rasgos léxicos en contexto.

Seguramente debido a que los alumnos de Acuicultura profundizaron su conocimiento sobre ciertos conceptos, pudieron relacionarlos con los temas de su especialidad. Lo que planteamos aquí es que, a mayor conocimiento del discurso de la especialidad, mayor es la cantidad, especificidad y seguridad de la información extraída.

Pudimos comprobar, asimismo, que cuanto mayor es el nivel lingüístico y discursivo en la L2, mejor es la comprensión de textos escritos. Los lectores eficaces utilizan pistas tanto semánticas como sintácticas, lo que refuerza la teoría de un modelo interactivo de procesamiento para la L2 en el que los lectores utilizan pistas a todos los niveles (Grabe, 2009). Podríamos hipotetizar sobre la existencia de un umbral, no solo lingüístico, sino posiblemente también temático, en este caso el conocimiento de la disciplina, ya que quizás en la interacción entre ambos componentes, más que en el efecto absoluto de uno solo, se encuentre la clave para la transferencia y la comprensión efectiva de los textos, lo que refuerza la idea del Marco Textual utilizado en esta intervención.

Es importante señalar que un número importante de investigaciones se ha focalizado 
en disciplinas como la medicina, las leyes, los negocios, la historia y en el ámbito de las organizaciones gubernamentales (Parodi, et al., 2010); no obstante, no existen estudios empíricos importantes en otras áreas del saber. Por esta razón, sería conveniente hacer más investigaciones que abarquen otras disciplinas y otros géneros académicos en diferentes instituciones del país y de Latinoamérica para corroborar los resultados obtenidos en este estudio. Finalmente, dado que el tamaño muestral es pequeño, no es posible generalizar los resultados a una población más amplia de estudiantes universitarios.

\section{Referencias}

Agencia de Calidad de la Educación (2013). Informe nacional de resultados SIMCE 2012. Santiago de Chile. Mineduc.

Agencia de Calidad de la Educación (2015). Síntesis Resultados de Aprendizaje SIMCE

Inglés 2014. Santiago de Chile: Mineduc.

Arancibia, R. (2010). Relaciones entre cantidad y calidad del conocimiento léxico y la comprensión de lectura en aprendientes de inglés como lengua extranjera. Tesis de Magister en Lingüística, mención Lengua Inglesa. Santiago, Chile: Universidad de Chile. Recuperado de http://www.repositorio.uchile. $\mathrm{cl} /$ handle/2250/108608

Barahona, M. (2014). Pre-service teachers' beliefs in the activity of learning to teach English in the Chilean context. Cultural-Historical Psychology, 10(2), 116-122.

Bazerman, C. (1988). Shaping Written Knowledge. Madison: The University of Wisconsin Press.

Bhatia, V. (2004). Worlds of written discourse. A genre based view. Sydney: Continuum.

Borg, S. (2006). Teacher cognition and language education. London, UK: Prentice Hall.

Díaz,C., Martínez, P., Roa, I., y Sanhueza, M. (2010). Una fotografía de las cogniciones de un grupo de docentes de inglés de secundaria acerca de la enseñanza y aprendizaje del idioma en establecimientos públicos de Chile. Folios, 31, 69-80.

Dörnyei, Z. (2003). Questionnaires in Second Language Research. New York: Lawrence Erlbaum Associates, Publishers.
Entwistle, N., McCune, V., and Hounsell, J. (2002). Approaches to studying and perceptions of university teaching-learning environments: Concepts, measures and preliminary findings, Occasional Report I. School of Education, University of Edinburgh, Paterson's Land, Holyrood Road, Edinburgh.

Grabe, W. E Stoller, F. L. (2002). Teaching and Researching Reading. London: Longman.

Grabe, W. (2009). Reading in a Second Language. Moving from Theory to Practice. New York: Cambridge University Press.

Gu, P. (2005). Learning strategies: Prototypical core and dimensions of variation. Recuperado de http://www. crie.org.nz/research-papers/Peter_Gu.pdf

Hernández, R., Fernández, C., y Baptista, P. (2006). Metodología de la Investigación. México: McGrawHill.

Hyland, K. (2006). English for Academic Purposes. An advanced resource book. London: Routledge Applied Linguistics.

Ibáñez, R. (2007). Comprensión de textos disciplinares escritos en inglés. RLA, 45(1), 67-85. Recuperado de http://dx.doi.org/10.4067/S071848832007000100005

Ibañez, R. (2008). Comprensión de textos académicos escritos en inglés: relación entre nivel de logro y variables involucradas. Revista Signos, 41(67), 203229.

Jiménez, P. (1998). Comprensión, estructura y dificultad. En Mario Bernales y Constantino Contreras (Organizadores), Por los caminos del Lenguaje (pp. 111-122). Temuco (Chile): Ediciones Universidad de la Frontera.

Johnston, C. (2001). Student perceptions of learning in first year in an economics and commerce faculty. Higher education Research and Development, 20(2), 169-184.

Kember, D., \& Wong, A. (2000). Implications for evaluation from a study of students'perception of good and poor teaching. Higher Education 40(1), 69-97.

Klassen, J., Lam, P., Chudley, P. \& Forde, K. (2013). Subject Teachers Teaching Learning Strategies: Two Case Studies. Recuperado de http://www.ugc.edu.hk/ tlqpr01/site/abstracts/057_klassen.htm

Koda, K. (2005). Insights into Second Language Reading. A Cross-Linguistics Approach. New York: Cambridge University Press.

Kozubíková, E., Petrusek,A., Diris,Z., Martín, MP., DiéguezUribeondo,J., \& Oidtmann, B. (2008). The old menace is back: Recent crayfish plague outbreaks 
in the Czech Republic. Aquaculture 274 (2-4), 208217. Recuperado de http://www.researchgate.net/ publication/223658728_The_old_menace_is_back Recent_crayfish_plague_outbreaks_in_the $\bar{C}_{-} \overline{\mathrm{C}} z e c{ }_{-}$ Republic

Lagos, M. (2011). La enseñanza de la comprensión lectora en inglés en la Universidad Católica de Temuco: un modelo de trabajo en el aula. Recuperado de http:// repositoriodigital.uct.cl/bitstream/handle/10925/181/ NST_0717-4268_03_2_art5.pdf?sequence $=1$

León, J. (2001). Las inferencias en la comprensión e interpretación del discurso. Un análisis para su estudio e investigación. Revista Signos 34(49-0), 113-125.

Martín, P. (2010). El inglés para fines académicos: aportaciones en la investigación y enseñanza del discurso científico. Revista de Lingüística y Lenguas Aplicadas, volumen 5, 109-121.

McKenzie, J. (1996, 8-12 July). Changes in university teachers'conception of teaching. Paper presented at the HERDSA Conference: Different Approaches: Theory and Practice in Higher Education, Perrth, Western Australia.

Mineduc (2009). Objetivos fundamentales y contenidos mínimos obligatorios de la educación Básica y Media. Actualización 2009. Santiago: Ministerio de Educación.

Neira, A., Reyes, F. y Riffo, B. (2015). Experiencia académica y estrategias de comprensión lectora en estudiantes universitarios de primer año. Literatura $y$ lingüística, 31, 221-244.

Recuperado de http://www.scielo.cl/scielo.php?script=sci arttextEpid $=$ S0716-

$58112015000100012 \varepsilon$ lng $=$ esEtlng $=$ es. S0716-58112015000100012

Oxford, R. L.and Crookall, D. (1989). Research on language learning strategies: Methods, findings and instructional implications. Modern Language Journal, 73, 404-419.
Oxford, R. (1990). Language learning strategies: what every teacher should know. Boston: Heinle \& Heinle.

Paris, S., Lipson, M. E Wixon, K. (1994). Becoming a strategic reader. En R.B. Ruddell, M. Ruddell y H. Singer (Eds.). Theoretical models and processes of reading, pp. 788 - 809. Newark: International Reading Association.

Parodi, G. (Coord.). Peronard, M. y Ibañez, R. (2010). Saber leer. Madrid: Aguilar.

Richards, J.C., Gallo, P., \& Renandya, W. (2001). Exploring teachers' beliefs and the processes of change. The PAC Journal, 1(1),41-62. Recuperado de http://www. pac- teach.org/jrnl-v1/

Sandelowski, M. (2003). Tables of tableaux? The challenges of writing and reading mixed methods studies. In A. Tashakkori and C. Teddlie (Eds.). Handbook of Mixed Methods in Social and Behavioral Research. Thousand Oaks, Calif.: Sage.

Sanhueza, G. y Burdiles, G. (2012). Diagnóstico de la competencia comunicativa en inglés de un grupo de escolares chilenos: puntos de encuentro con su perfil estratégico. Folios, 36, 97-113.

Schuh, K. L., Walker, S., Kizzie, J. E., \& Mohammed, M. (2001). Perturbation and reflection as tools of change in beliefs: the struggle with lecture. The University of lowa, lowa City.

Swales, J. (1990) Genre analysis: English in academic and research settings. Cambridge:

Cambridge University Press.

Taylor, P. (1996). Reflections on students'conception of learning and perceptions of learning environments. Higher Education Research and Development, 15(2), 223-237.

Vigotsky, L. (1988). El desarrollo de los procesos psicológicos superiores. México: Editorial Crítica, Grupo Editorial Grijalbo. 